\title{
Progress in Superconducting Quantum Computation: Quantum Simulation of Dynamical Phase Transitions
}

Q

rantum simulation uses a controllable quantum system to mimic complex systems or solve intractable problems, among which the nonequilibrium problems of quantum many-body systems have attracted wide research interest since they are hard to simulate using classical computers. Instead, popular quantum simulators, such as superconducting circuits. can provide insights into these problems. As considerable advances have been made in scalability, coherence and controllability. superconducting circuits have become one of the most important quantum simulation platforms.

Recently, a research group joining scientists from the Institute of Phrsics (IOP) of Chinese Academy of Sciences (CAS) and Zhejiang University in China. and RIKEN in Japan, have successfully performed quantum simulation of dynamical phase transitions in a LipkinMeshkov-Glick model with a 16-qubit superconducting quantum simulator:

Dynamical phase transition (DPT) is a kind of nonequilibrium phase transitions and have been theoretically investigated in various quantum many- body models. There are two types of DPT. The first type of DPT (DPT-1) focuses on the non-equilibrium order parameter, while the second type of DPT (DPT2 ) is characterized by the non-analvtical behaviors of Loschmidt echo associated with the Lee-Yang-Fisher zeros in statistical mechanics. Further theoretical and numerical investigations reveal that the DPT-1 and DPT-2 can be studied in the same framework.

The quantum processor integrates 20 superconducting qubits which are all coupled to a common resonator bus. which was used to generate Schrödinger cat states in previous work. This time. 16 qubits are used to engineer the Iipkin-MeshkovGlick (LMG) model by applying controllable transverse field to each qubit. The system is driven into a nonequilibrium state with microwaves and then evolve under the LMG model. Firstly, typical features of the DPT-1 are observed (Fig. 1). For small transverse field, the system stays in the dynamical ferromagnetic phase (DFP), and the magnetization exhibits a slow relaxation. However. given a strong transverse field. the system enters into the dynamical paramagnetic
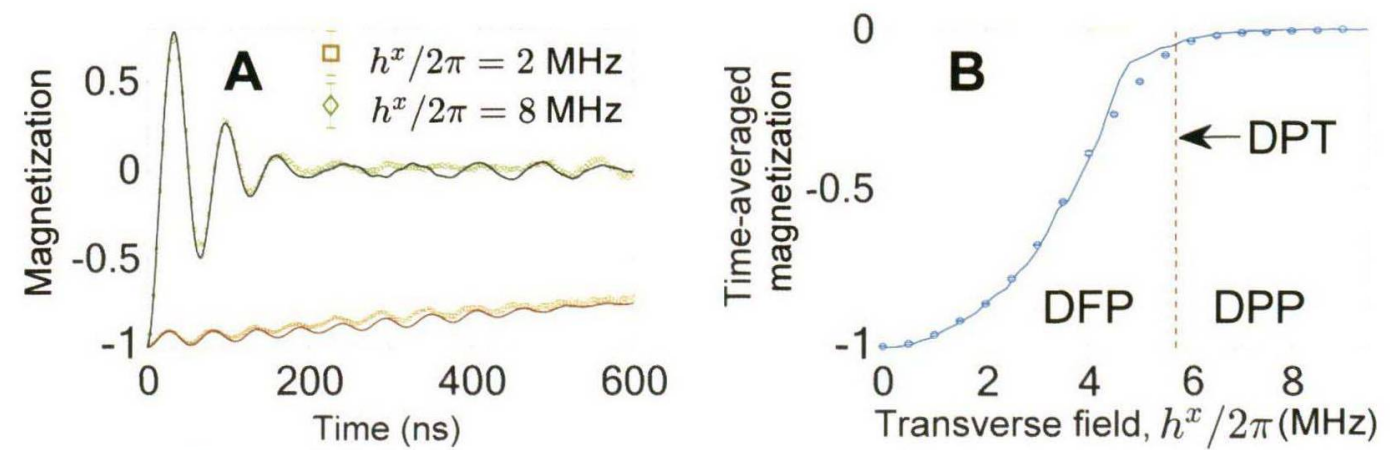

Fig. 1 Time evolution of the magnetization and time-averaged magnetization as a function of transverse field. 


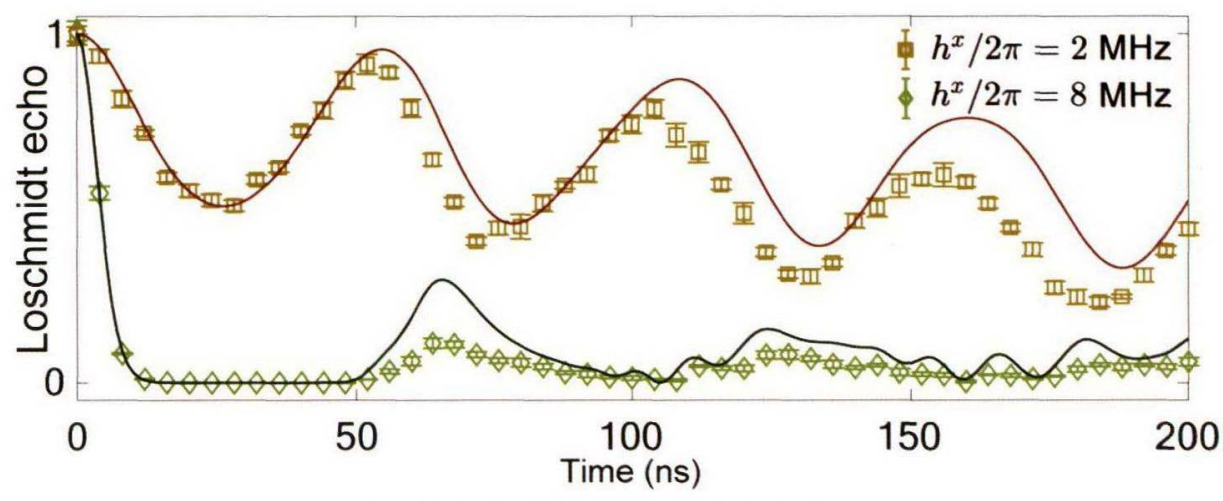

Fig. 2 Time evolution of the Loschmidt echo.

phase (DPP), and the magnetization exhibits a large oscillation at an early time and approaches zero in the long-time limit. The time-averaged magnetization as the non-equilibrium order parameter is zero in the DPP. while it becomes finite in the DFP. Next, the researchers demonstrate that there exist the zeros of Loschmidt echo in the DPP, suggesting the relation between DPT-1 and DPT-2 (Fig. 2).

In addition, the researchers explore the DPTs from a novel perspective. They study the minimum spin squeezing as a probe of the phase transition. The experimental results show that the minimum value of the spin-squeezing parameters can be attained very close to the critical point of the DPT. This indicates a potential application of the DPT to quantum metrology.

This study entitled "Probing dynamical phase transitions with a superconducting quantum simulator" was published on Science Advances.

The study was supported by the National Basic Research Program of China, the National Natural Science Foundation of China, the Strategic Priority Research Program of the Chinese Academy of Sciences,

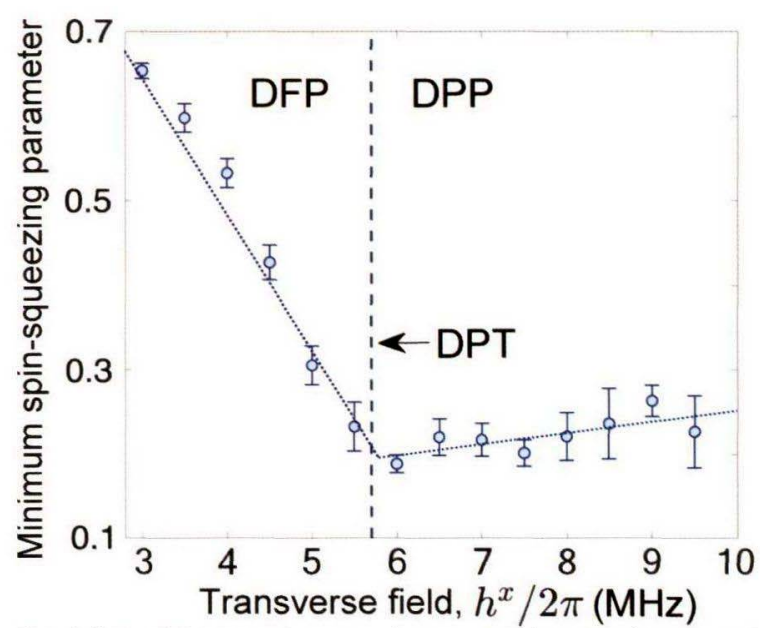

Fig. 3 The minimum spin-squeezing parameter as a function of transverse field.

the China Postdoctoral Science Foundation and grants from Japan.

\section{Contact:}

FAN Heng

Institute of Physics, CAS

Email: hfan@iphy.ac.cn 